\title{
Investigation of the influence of the geometrical dimensions of the striker on the efficiency of energy transfer of shock pulses during wave strain hardening
}

\author{
Andrey Kirichek ${ }^{1, *}$, Sergey Barinov ${ }^{2}$, and Aleksandr Yashin $^{2}$ \\ ${ }^{1}$ Vice Rector for Prospective Development, BSTU, 241035 Bryansk, Russia \\ ${ }^{2}$ MET Department, VLSU, 600000 Vladimir, Russia
}

\begin{abstract}
The article presents data on the influence of the geometric parameters of a flat-faced rod shock system on the efficiency of a shock pulse energy transfer to the deformation zone during wave strain hardening. As a result of the simulation, it was found that an increase in the ratio of the striker length to its diameter from 0.1 to 10 allows transferring 15.6 times more shock pulse energy to the deformation area. Changing the ratio of the lengths of the striker and the waveguide from 0.1 to 10 increases the share of the transmitted shock pulse energy by 2.8 times.
\end{abstract}

\section{Introduction}

The modern level of technology urgently requires more and more reliability and service life from machine parts, which are ensured by the creation of materials of new generations and new technologies for their hardening. One of the most promising directions in the development of modern hardening technologies is the formation of a heterogeneous structure of the modified surface layer of a continuous non-gradient material, which, on the one hand, does not have pronounced boundaries of zones with changed properties, and, on the other hand, is similar in properties to a composite material with a soft matrix and solid inclusions.

Wave strain hardening (WSH) is the only technology that makes it possible to use the surface plastic deformation method to create a multilayer heterogeneous, naturally reinforced structure in the product surface layer [1-3]. The location of such structures at a depth of $6-10 \mathrm{~mm}$ can increase the operational properties of machine parts by 3-6 times [1$3]$. The technology of wave strain hardening is interesting for modern industry, since it allows us to increase the payload on the material, and thereby open up huge reserves for improving the tactical and technical characteristics of products in aviation, astronautics, automotive, general engineering, tool manufacturing, energy, oil and gas and construction industries.

The technology is based on a shock through an intermediate link - a waveguide, due to which a deformation wave is formed. A deformation wave is a stream of pulses

\footnotetext{
* Corresponding author: avk@tu-bryansk.ru
} 
characterized by the presence of not only the head but also the tail, which is formed due to the reflection and superposition of elastic waves in the striker, waveguide, and hardened product. This makes it possible to increase the pulse duration by 10 times, the efficiency of impact energy by several times. Previously performed studies were carried out in a rather narrow range; nevertheless, a high dependence of the pulse duration on the parameters of the applied shock system was revealed. It is generally accepted that the following ratios of the lengths and areas of the cross sections of the striker and waveguide are most effective for WSH: $L_{1} / L_{2}=3 ; d_{1} / d_{2}=1 \ldots 3[1]$.

The process of wave strain hardening can be controlled by a large number of technological factors in a wide range of values. On the one hand, this allows a rather fine control of the WSH process on the other hand, due to the large amount of experimental data, difficulties arise in establishing relationships between technological factors that ensure the formation of the required strengthening parameters.

The study of the influence of a large number of WSH controlled technological factors of on the hardening efficiency is easy to carry out in the modeling process. The existing analytical model of pulse formation in the deformation area, wave transformation at the boundaries of zones with various acoustic properties, as well as a computer program created on its basis, require rather cumbersome calculations and are based on assumptions that must be clarified with the FEM (Finite Element Model) [1]. So, physical and mechanical properties of the processed material are considered semi-experimentally, without taking into account hardening curves; hardening is carried out with only one tool; hardness (stress, strain) formed in the loading medium as a result of the calculation can be determined only at one point, under the imprint from the impact of the tool, and not by the equidistant surface of the material. Although finite-element models proposed later by $\mathrm{A}$. N. Afonin [4] allow us to identify the nature of the relationship between the fields of stresses, strains, design and technological parameters of the thread rolling process with static and static-pulse loading of a deforming tool, they have a narrow focus and are intended only for solving problems related to thread rolling. Therefore, the task of developing a comprehensive model of the wave strain hardening process of a modified layer, which allows us to get rid of the limitations of earlier models, is relevant.

The aim of this paper is to identify the general patterns of the WSH process, to search for the conditions of the most efficient transfer of shock pulse energy to the deformation zone by means of finite element modeling.

\section{Materials and methods}

Most modern modeling means are based on the finite element method [5-7]. The leader in the field of modeling short-term transient processes $\left(<10^{-5} \mathrm{~s}\right)$ of the contact interaction of several bodies is the LS-DYNA software module. The finite element model of the shock pulse formation consists of the shock system of the striking waveguide (cylindrical rods with flat ends) and a loaded medium (Figure 1). The flat end of the waveguide is preliminarily statically pinched $P_{s t}$ to the loading medium. The striker and waveguide material is $90 \mathrm{CrSi}$ hardened steel. The material of the loading medium is $41 \mathrm{Cr} 4$ steel in the delivery state. In the model the striker delivers a single coaxial strike $P_{i}$ against the waveguide with an energy of $35 \mathrm{~J}$. The diameters of the striker $d_{1}$ and the waveguide $d_{2}$ were not changed to achieve the objectives of the experiment; they were taken equal to 60 $\mathrm{mm}$. The magnitude of the shock pulse energy reported to the deformation area is evaluated by the method described in the source [1]. The efficiency of the process was estimated by the value of the energy transfer coefficient $\eta$, which is equal to the ratio of the pulse energy in the deformation area to the impact energy of the striker on the waveguide. The influence of the geometric dimensions of the striker and the waveguide on the transfer of the shock 
pulse energy to the deformation area is estimated, it is assumed that the tool is the end of the waveguide.

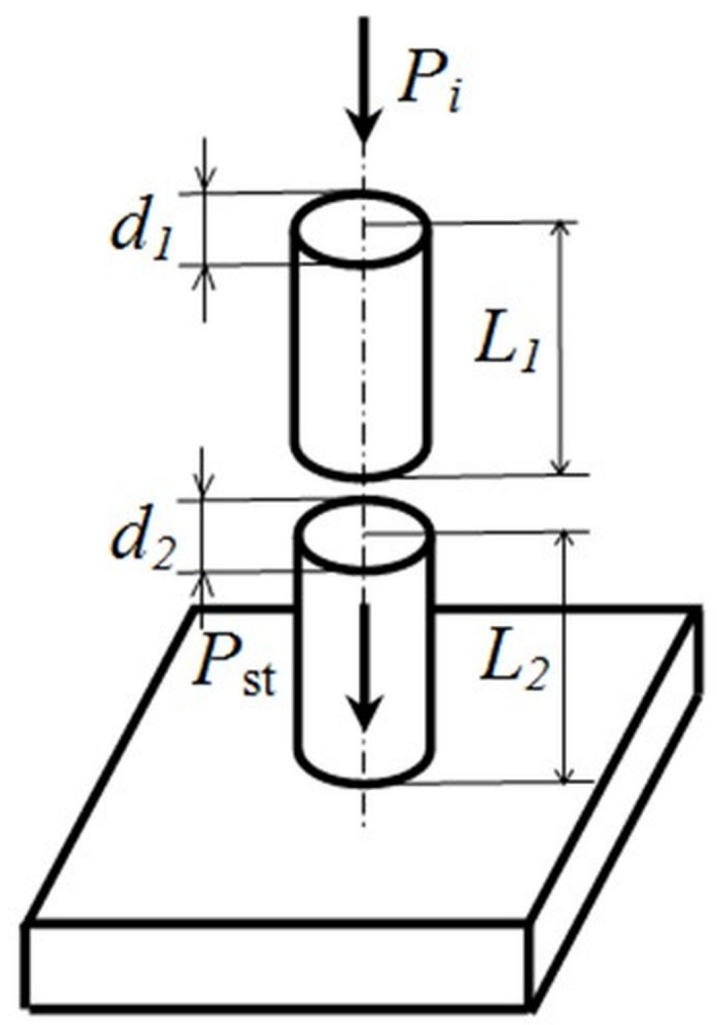

Fig. 1. Scheme of the shock system.

According to previous studies, the geometric parameters of the striker and waveguide have a significant effect on the energy transfer efficiency in a rod shock system $[8,9]$. Based on this, shock systems are simulated with the ratio of the $L_{1}$ striker lengths and the $L_{2}$ waveguide in the range of $0.1<L_{1} / L_{2}<10$.

Previously, insufficient attention has been paid to the study of the effect of the ratio of the lengths of the striker $L_{l}$ and its diameter $d_{l}$ on the value of the transferred energy of the shock pulse to the deformation area at WSH. For this purpose, models of shock systems have been created that make it possible to establish the effect of the ratio of the sizes of the lengths of the striker $L_{l}$ and its diameter $d_{l}$ on the value of the transmitted energy of the shock pulse in the deformation zone, in the range of $0.1<L_{1} / d_{l}<10$. In connection with the change in the mass of the striker (in order to maintain the given ratios $L_{l} / L_{2} ; L_{I} / d_{1}$ ), to ensure the constancy of the impact energy $\left(P_{i}=35 \mathrm{~J}\right)$, the impact velocity also changes.

The adequacy of the obtained model of a shock pulse formation with WSH was estimated by comparing it with the experimental data obtained at the shock system bench model. The strength, duration, and energy of the shock pulse in the deformation area obtained from the finite element model correspond to the experimental results with a confidence level of 0.95 . 
3 The study of the influence of the geometrical dimensions of the striker and the waveguide on the efficiency of energy transfer of shock pulses energy transfer with wave strain hardening

3.1 The study of the influence of the ratio of the $L_{1}$ striker length to its diameter $d_{1}$ on the efficiency of the energy of shock pulses transmitted by the shock system to the deformation zone

A graphical interpretation of the results of the studies is presented in Figure 2.

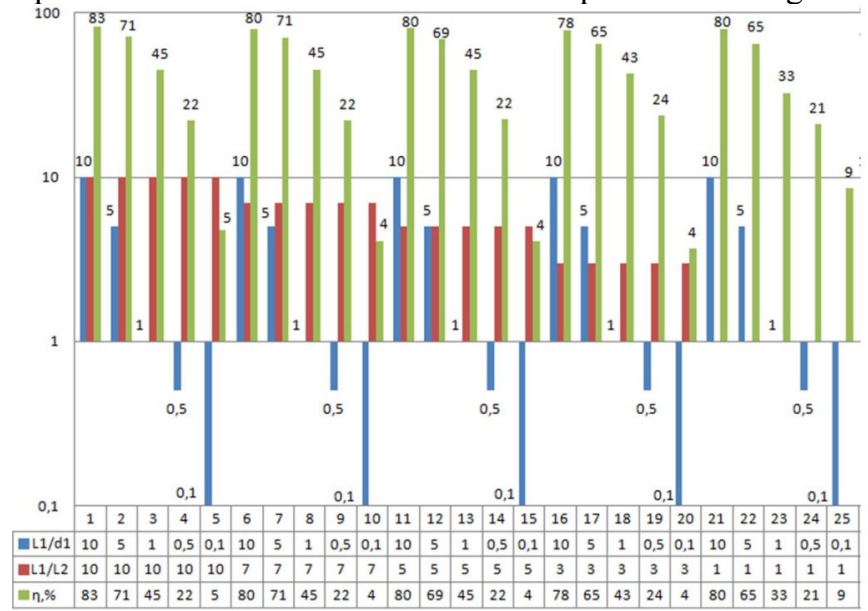

Fig.2. Beginning.

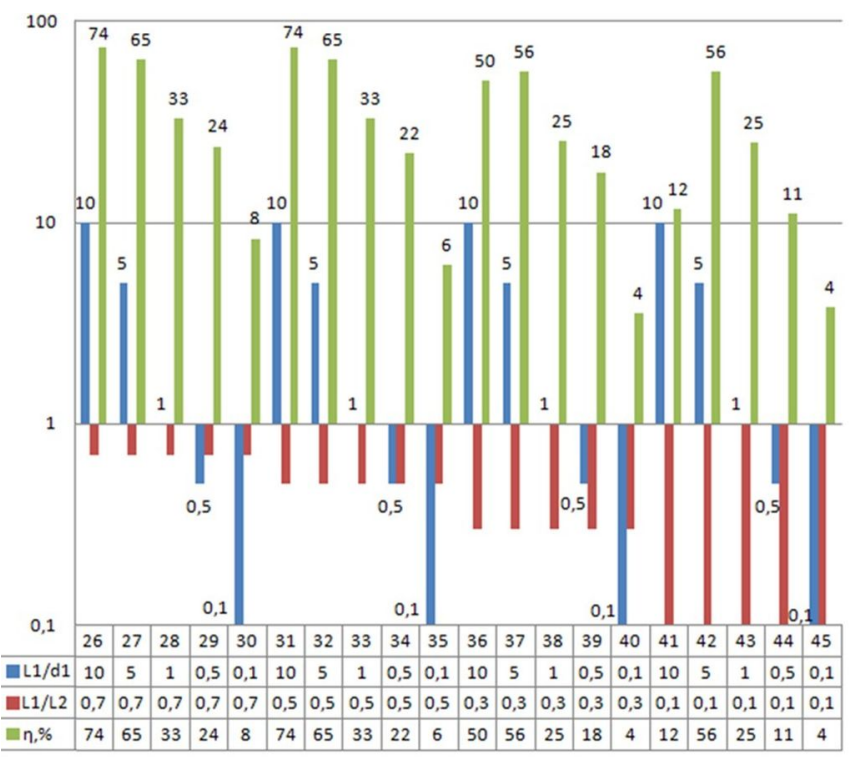

Fig. 3. Diagram of the dependence of the fraction of the transmitted energy of the shock pulse in the deformation zone on the ratio of the shock system parameters $L_{1} / d_{1}$ and $L_{1} / L_{2}$. 
The smallest fraction of the energy (4-9\%) transferred to the deformation was established at the $L_{l} / d_{l}=0.1$ ratio.

With an increase in the $L_{1} / d_{1}$ ratio to 0.5 , the proportion of transmitted energy increased to $21-24 \%$. The worst growth of $\eta$ to $11-18 \%$ was found in the $0.1 \leq L_{1} / L_{2} \leq 0.3$ shock systems.

With an increase in the $L_{1} / d_{1}=1$ ratio, the largest value (of $43-45 \%$ ) of the fraction of the transmitted energy in the deformation zone was noted in shock systems with $3 \leq L_{1} / L_{2}$ $\leq 10$. $0.1 \leq L_{1} / L_{2} \leq 1$ impact systems showed a smaller increase of $\eta$ to $25-33 \%$.

An increase in $L_{l} / d_{1}$ to 5 led to $65-71 \%$ increase in the fraction of transmitted energy, which is more than at $L_{1} / d_{1}=1$ by $51-57 \%$.

An increase in the $L_{1} / d_{1}$ ratio to 10 showed the maximum possible increase in the fraction of the transmitted energy in the deformation area to $80-83 \%$, in shock systems, where $0.5 \leq L_{1} / L_{2} \leq 10$, which is $17-23 \%$ more than at $L_{1} / d_{1}=5$. However, with $L_{1} / d_{1}=10$, for $0.1 \leq L_{1} / L_{2} \leq 0.3$ shock systems, a decrease of $\eta$ by 4.6 and 1.12 times, respectively, was noted.

To assess the possibilities of increasing the fraction of transmitted energy to the deformation zone by increasing the $L_{l} / d_{1}$ ratio, the range of $L_{l} / d_{l}$ values was increased to 20-30, for shock systems with $L_{1} / d_{1}=10$. As a result, the obtained $\eta$ values did not change and amounted to $82-83 \%$. The possibility of transferring a fraction of energy close to unity in rod shock systems with flat ends was noted by V.E. Eremjants in his works [8,9]. The obtained simulation results are fully consistent with the theoretical concepts of the formation of deformation waves in shock systems.

\subsection{The investigation of the influence of the ratio of the $L_{1}$ striker lengths and the $L_{2}$ waveguide on the efficiency of the energy of shock pulses transmitted by the shock system to the deformation area}

An analysis of the effect of the ratios of the $L_{1}$ striker lengths and the $L_{2}$ waveguide on the efficiency of the shock pulse energy transfer to the deformation area revealed that, with the $L_{1} / d_{1}=0.1$ ratio, a change of $0.1 \leq L_{1} / L_{2} \leq 1$ is accompanied by an increase in $\eta$ from 4 to $9 \%$.

At $L_{1} / d_{1}=0.5$, the largest increase in $\eta$ from 11 to $22 \%$ was observed at $0.1 \leq L_{1} / L_{2} \leq 0.5$. With an increase of $0,7 \leq L_{1} / L_{2} \leq 10$, the share of the transmitted energy in the deformation zone did not change and amounted to $21-24 \%$.

At $L_{1} / d_{1}=1$, an increase in $L_{1} / L_{2}$ from 0.3 to 0.5 increased $\eta$ from 25 to $33 \%$. A further increase in $\eta$ was achieved with an increase in $L_{1} / L_{2}$ from 1 to 3 and amounted to 33 and $45 \%$ respectively.

At $L_{1} / d_{1}=5$, an increase in $L_{1} / L_{2}$ from 0.3 to 0.5 led to an increase in $\eta$ from 56 to $65 \%$. A further increase in the fraction of the transmitted energy of the shock pulse in the deformation area from 65 to $71 \%$ was achieved with a change in $L_{1} / L_{2}$ from 3 to 10 .

At $L_{1} / d_{1}=10$, a change in $L_{1} / L_{2}$ from 0.1 to 0.3 is accompanied by an increase in $\eta$ from 12 to $50 \%$. An increase in $L_{1} / L_{2}$ from 0.3 to 0.5 is accompanied by an increase in $\eta$ from 50 to $74 \%$. Further growth of $L_{1} / L_{2}$ from 1 to 10 leads to an increase in $\eta$ from 74 to $83 \%$.

\section{Conclusion}

1. It is established that in rod shock systems with flat ends, in the range of ratios of $0.1 \leq$ $L_{1} / L_{2} \leq 10 ; 0.1 \leq L_{1} / d_{1} \leq 10$ at $d_{1}=d_{2}=60 \mathrm{~mm}$, the largest share of the shock pulse energy transferred to the deformation zone is $83 \%$. This maximum value was obtained at $L_{l} / d_{1}=$ $10 ; L_{1} / L_{2}=10$ 
2. It is established that an increase in the $L_{l} / d_{l}$ ratio to $20 \ldots 30$ does not lead to a further increase in the fraction of the shock pulse energy entering the deformation area. In addition, the use of $L_{1} / d_{1}$ more than 10 in most cases, non-technological and inappropriate.

3 . It is established that an increase in the ratio of the striker length $L_{l}$ to its diameter $d_{l}$ with $0.1 \leq L_{1} / d_{1} \leq 10$ allows, on average, to increase the fraction of the shock pulse energy transmitted to the deformation zone by 15.6 times. An increase in the ratio of the lengths of the striker $L_{1}$ and the waveguide $L_{2}$ with $0.1 \leq L_{1} / L_{2} \leq 10$ allows us, on average, to increase the fraction of the shock pulse energy transmitted to the deformation zone only 2.8 times.

The reported study was funded by RFBR according to the research project No. 18-38-20066

\section{References}

1. A.V. Kirichek, D.L. Soloviev, A.G. Lazutkin, Technology and equipment for staticpulse treatment by surface plastic deformation, Mech. Eng., 288 (2004)

2. A.V. Kirichek, S.V. Barinov, A.V. Yashin, Key Eng. Mater., 736, 12 (2017)

3. A.V. Kirichek, S.V. Barinov, A.V. Aborkin, A.V. Yashin, A.A. Zaicev, IOP Conf. Series: Mater. Science and Eng., 327, 042011 (2018)

4. A.V. Kirichek, A.N. Afonin Proceed. 2010 Joint China-Russia Sympos. on Adv. Mat. and Proc. Tech., Harbin, 137 (2010)

5. G. Strang, G. J. Fix, An Analysis of the Finite Element Method (Prentice-Hall, 1973)

6. E.G. Thomsen, T.Y. Charles, S. Kobayashi, Mechanics of plastic deformation in metal processing, 486 (1965)

7. S. Kobayashi, S. Oh, T. Altan, Metal Forming And The Finite Element Method (Oxford University Press, New York, 1989)

8. V.E. Eremjants, V.V. Niu, Mod. prob. of theory of mash, 4, 123 (2016)

9. V.E. Eremjants, V.V. Niu, Journ. of Adv. Res. in Tech. Science, 2, 20 (2016) 